\title{
Perfil epidemiológico de puérperas de recém- -nascidos com baixo peso e prematuros
}

\section{Epidemiological profile of low-weight and prematured newborn puerpers}

Silas Santos Carvalho ${ }^{1}$ Julita Maria Freitas Coelho II

'Universidade Estadual de Feira de Santana (UEFS), Feira de Santana/ "I Professora na Faculdade Anísio Teixeira e da Universidade Estadual de Feira de Santana.

BA - Brasil.
RESUMo O baixo peso ao nascer (BPN) constitui um dos fatores determinantes da probabilidade do recém-nascido (RN) sobreviver ao período neonatal, e, paralelo à prematuridade, decorre de circunstâncias diversas e imprevisíveis, associadas a condições socioeconômicas maternas desfavoráveis. O objetivo deste estudo é descrever o perfil epidemiológico de puérperas de recém-nascidos com baixo peso e prematuros. Trata-se de um estudo caso-controle com 144 puérperas num hospital público de Feira de Santana-BA. O grupo caso $(n=24)$ foi constituído por mães de nascidos vivos com baixo peso $(<2500 \mathrm{~g})$ e $<37$ semanas, e o grupo controle $(n=120)$, por mães de nascidos vivos com peso normal $(\geq 2500 \mathrm{~g})$ e $\geq 37$ semanas. Foram realizadas aplicação de um questionário e consulta de prontuários e cartão pré-natal. Os principais fatores de risco para o BPN e prematuridade foram avaliados mediante análise bivariada e obtenção da medida de associação odds ratio (OR). Os principais resultados demonstraram que $16,7 \%$ das gestantes tiveram filhos com BPN e prematuros, sendo que a maioria destas tinha faixa etária entre 14 anos e 25 anos, cor negra ou parda, teve parto normal, consumiu bebida alcoólica durante a gestação e fez menos de seis consultas de pré-natal. A identificação dos fatores de risco do BPN é relevante para a saúde pública, possibilitando subsídios para o planejamento de estratégias de enfrentamento desse agravo.

Palavras-chave: Gravidez. Baixo peso ao nascer. PrematuridaDE. FATORES DE RISCO.

Abstract Low birth weight (LBW) is one of the determinants of the probability of surviving the neonatal period and, parallel to prematurity, is due to diverse and unpredictable circumstances associated with unfavorable maternal socioeconomic conditions. The objective of this study is to describe the epidemiological profile of puerperae of low birth weight and preterm infants. This is a case-control study with 144 puerperae in a public hospital in Feira de Santana-BA. $(<2500 \mathrm{~g})$ and $<37$ weeks, and the control group $(\mathrm{n}=120)$ with mothers of live births with normal weight $(>2500 \mathrm{~g})$ and $>37$ weeks. Applications of questionnaires and records and prenatal consultations were performed. The main risk factors for BPN and prematurity for analysis through bivariate analysis and obtaining the odds ratio (OR) measure. The main results showed that $16.7 \%$ of the pregnant women had children with LBW and preterm infants. Most of the styles were between 14 and 25 years old, black or brown, had a normal birth, consumed alcohol during pregnancy and did less of six prenatal visits. The identification of BPN's risk risks is relevant to public health, allowing for subsidies for the planning of strategies to address the problem.

Key-words: Pregnancy. Low weight at birth. Prematurity. EpiDEMIOLOGY. Risk factors. 


\section{INTRODUÇÃO}

O perfil epidemiológico da puérpera e de seu recém-nascido ( $\mathrm{RN})$ tem sido considerado um instrumento para a obtenção de dados a fim de contribuir no planejamento de ações para proporcionar melhorias na qualidade da atenção destinada a esse grupo. Há situações em que o desfecho de uma gestação não é um RN sadio com mínimo de trauma para a puérpera, devido à complicações durante a gravidez ou parto, ou com o concepto. A compreensão da epidemiologia de uma população fornece a integralidade do cuidado e a eficácia do seu atendimento, devido às inúmeras variáveis que interferem no processo saúde-doença. ${ }^{1,2}$

$\mathrm{O}$ peso ao nascer constitui um dos fatores determinantes da probabilidade de sobreviver ao período neonatal. O RN com peso inferior a 2.500 gramas é classificado como baixo peso ao nascer (BPN). Essa condição leva a um maior risco de infecções, propensão ao retardo de crescimento e déficit neuropsicológico podendo repercutir na fase adulta. ${ }^{3,4}$

Embora se acredite que os fatores condicionantes e determinantes do BPN se inter-relacionem de forma complexa e se originam de condições biológicas, sociais e ambientais às quais a mulher está exposta durante a gestação, ainda faltam achados mais consistentes sobre os determinantes para esse agravo. ${ }^{5-7}$

A prematuridade é definida como a gestação concluída entre a $20^{\mathrm{a}}$ e a $37^{\mathrm{a}}$ semanas ou entre 140 e 257 dias após o primeiro dia da última menstruação. ${ }^{8}$ Ela decorre de circunstâncias diversas e imprevisíveis, comprometendo custo social e financeiro à so- ciedade em geral, o que demanda, também, capacidade técnica e equipamentos nem sempre disponíveis na estrutura assistencial, requerendo cuidados de maior nível de complexidade. ${ }^{9,10}$

Segundo Cruz et al. ${ }^{5}$, a ocorrência de BPN e prematuridade está associada a condições socioeconômicas desfavoráveis, como nutrição inadequada, baixo índice de massa corpórea (IMC) pré-gestacional, extremos de idade, hábito de fumar e alcoolismo durante a gravidez, primiparidade e complicações no período gestacional.

Diante do exposto, surge a necessidade de melhor compreender os mecanismos e fatores de risco que se relacionam diretamente com o aparecimento do BPN e prematuridade. Dessa forma, o presente estudo tem o objetivo de descrever o perfil epidemiológico de puérperas de recém-nascidos com baixo peso e prematuros.

\section{MÉTodos}

Estudo caso-controle (BPN e Controles), no qual foram avaliados dados referentes às mulheres atendidas num hospital público de Feira de Santana, município do interior da Bahia, para a realização de parto no período compreendido entre junho e agosto de 2015.

Foram incluídas na pesquisa todas as gestantes que compareceram ao hospital para realizar o parto, no período supracitado, e que tinham a idade gestacional e o peso ao nascer do bebê disponíveis nos registros de nascimento do hospital e nos prontuários. O grupo caso foi composto por 24 mães de $\mathrm{RN}$ com $<2500 \mathrm{~g}$ e com idade gestacional $<37$ semanas e o grupo controle por 120 
mães de RN com peso igual ou superior a $2500 \mathrm{~g}$ e com idade gestacional $\geq 37$ semanas. Todas que aceitaram participar do estudo assinaram o termo de consentimento livre e esclarecido, bem como demais condutas éticas previstas na Resolução 466/2012 do Conselho Nacional de Saúde, ${ }^{11}$ que trata de pesquisa envolvendo seres humanos.

Para o cálculo da IG (semanas completas), foram usadas a data da última menstruação (DUM) e a informação da ultrassonografia (USG), disponível no cartão, sendo a USG preferida apenas quando realizada com IG $\leq 12$ semanas, havendo discordância maior que uma semana entre as IG obtidas com base na DUM e na USG. Na ausência dessas duas informações, foi utilizada a IG registrada pelo pré-natalista e na indisponibilidade dessa terceira informação, a gestante foi excluída da análise.

Foi utilizado um questionário que incluiu dados sociodemográficos, cuidados com a saúde geral, história gestacional, qualidade do pré-natal e hábitos de vida. Informações complementares foram coletadas em prontuários e no cartão de pré-natal. Não foram incluídas no estudo as puérperas com internamento hospitalar pós-parto superior a sete dias.

Os dados foram tabulados no programa Statistical Package for Social Science (SPSS) 17.0 e analisados no programa STATA, versão 10.0 e versão 11.0. Para a avaliação das associações, foi realizada análise estratificada para a obtenção das medidas brutas de Odds Ratio (OR). Para avaliar o grau de homogeneidade ou comparabilidade entre os grupos, foi empregado o Teste ${ }_{\mathrm{x}}{ }^{2}$ (Qui-Quadrado) de Pearson e o Teste Exato de Fischer quando a frequência esperada foi menor do que 5, com nível de significância de $5 \%$ e Intervalo de Confiança (IC) de 95\% $(\mathrm{p}<0,05)$.

A pesquisa foi aprovada pelo Comitê de Ética em Pesquisa da Faculdade Anísio Teixeira (FAT) de Feira de Santana-BA (Parecer 1.078.653). Não há conflito de interesse em relação aos métodos utilizados como parte da investigação ou interesse financeiro dos pesquisadores.

\section{RESUlTADOS}

Da amostra selecionada $(\mathrm{n}=144), 24$ $(16,7 \%)$ puérperas compuseram o grupo caso (mães de RN com peso inferior a $2500 \mathrm{~g}$ e com idade gestacional abaixo de 37 semanas), e 120 (83,3\%) puérperas o grupo controle, composto por mães de RN com peso igual ou superior a $2500 \mathrm{~g}$ e com mais de 37 semanas de gestação (Tabela 1).

Entre as mulheres do grupo caso, observou-se predominância de 14 anos a 25 anos de idade $(58,3 \%)$, de raça/cor de pele parda $(87,5 \%)$, com oito ou mais anos de estudo $(95,8 \%)$, que tiveram parto normal $(62,5 \%)$, já tinham tido um ou mais partos $(54,2 \%)$, tinham um estado nutricional pré-gestacional (IMC $\geq 25$ e $<30 \mathrm{~kg} / \mathrm{m}^{2}$ ) $(58,3 \%)$, eram etilistas $(50 \%)$ e tomaram medicamento abortivo $(16,7 \%)$. Houve relato de hiperêmese (12), parasitoses (10) e pré-eclâmpsia (5) em 18,8\% das gestantes, sendo que $33,3 \%$ destas tiveram RN com $\mathrm{BPN} /$ prematuro. Além disso, tiveram condições menos favoráveis no tocante ao número de consultas de pré-natal menor que seis $(58,3 \%)$, infecção urinária $(37,5 \%)$ e ocupação (25\%) (Tabela 1). 
Tabela 1 - Características demográficas, socioeconômicas, obstétricas, de saúde geral e de estrutura do pré-natal de puérperas dos grupos Controles e BPN (n=144). Feira de Santana-BA, 2015.

\begin{tabular}{|c|c|c|c|c|c|c|}
\hline \multirow[t]{2}{*}{ Variáveis } & \multicolumn{2}{|c|}{ BPN } & \multicolumn{2}{|c|}{ Controles } & \multirow[t]{2}{*}{ OR } & \multirow[t]{2}{*}{$\mathbf{p}^{*}$} \\
\hline & $\mathrm{n}=\mathbf{2 4}$ & $\%$ & $\mathrm{n}=120$ & $\%$ & & \\
\hline \multicolumn{7}{|l|}{ Idade (em anos) } \\
\hline $14-25$ & 14 & 58,3 & 89 & 74,2 & 1,00 & \\
\hline $26-47$ & 10 & 41,7 & 31 & 25,8 & 1,86 & $0,36^{\ddagger}$ \\
\hline
\end{tabular}

\section{Raça/Cor Materna}

Branca/amarela

03

12,5

05

87,5

115

4,2

1,00

Negra/parda

21

\section{7,5}

Nível de escolaridade

(anos estudo)

$0-3 \quad 0$

$\geq 4$

23

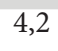

10

8,3

1,42

$1,00^{* *}$

Renda (salário mínimo)

$<1$

Ocupação Materna durante

a Gestação

Empregada

Desempregada

Tipo de parto

Normal

Cesárea

09

95,8

110

91,7

1,00

\section{Primiparidade}

Sim

11

45,8

56

20,9

79,1

0,91

1,00

Não

13

54,2

64

46,7

0,90

$0,84^{\ddagger}$

Histórico de Nascidos com

\section{Baixo Peso}

Sim

Não

04

20

16,7

10

8,3

$2,78 \quad 1,00^{* *}$

Histórico de Nascidos

\section{Prematuros}

Sim

01

83,3

110

91,7

1,00

Não

23

4,2

07

5,3

0,70

$0,25^{* *}$

Uso de tabaco durante a

gestação

Sim

$$
01
$$

4,2

11

9,2

$2,2 \quad 0,94^{\star *}$

Não

23

95,8

109

90,8

1,00 


\begin{tabular}{|c|c|c|c|c|c|c|}
\hline \multirow[t]{2}{*}{ Variáveis } & \multicolumn{2}{|c|}{ BPN } & \multicolumn{2}{|c|}{ Controles } & \multirow[t]{2}{*}{ OR } & \multirow[t]{2}{*}{$\mathbf{p}^{*}$} \\
\hline & $\mathrm{n}=\mathbf{2 4}$ & $\%$ & $\mathrm{n}=120$ & $\%$ & & \\
\hline \multicolumn{7}{|c|}{$\begin{array}{l}\text { Uso de álcool durante a } \\
\text { gestação }\end{array}$} \\
\hline Sim & 12 & 50,0 & 36 & 30,0 & 7,9 & $<0,01^{*}$ \\
\hline Não & 12 & 50,0 & 84 & 70,0 & 1,00 & \\
\hline \multicolumn{7}{|l|}{$\begin{array}{l}\text { No. de Consultas no } \\
\text { Pré-Natal }\end{array}$} \\
\hline$<6$ & 14 & 58,3 & 44 & 36,7 & 2,94 & $0,02^{\star}$ \\
\hline$\geq 6$ & 10 & 41,7 & 76 & 63,3 & 1,00 & \\
\hline \multicolumn{7}{|l|}{$\begin{array}{l}\text { Tomou medicamento } \\
\text { abortivo }\end{array}$} \\
\hline Sim & 04 & 16,7 & 05 & 4,2 & 5,05 & $0,04^{*}$ \\
\hline Não & 20 & 83,3 & 115 & 95,8 & 1,00 & \\
\hline \multicolumn{7}{|l|}{ Infecção urinária } \\
\hline Sim & 09 & 37,5 & 49 & 40,8 & 0,98 & $0,96^{\ddagger}$ \\
\hline Não & 15 & 62,5 & 71 & 59,2 & 1,00 & \\
\hline \multicolumn{7}{|l|}{ Hipertensão } \\
\hline Sim & 04 & 16,7 & 12 & 10,0 & 2,11 & $0,42^{\star *}$ \\
\hline Não & 20 & 83,3 & 108 & 90,0 & 1,00 & \\
\hline \multicolumn{7}{|l|}{ Outras doenças } \\
\hline Sim & 10 & 41,7 & 29 & 24,2 & 0,76 & 0,06 \\
\hline Não & 14 & 58,3 & 91 & 75,8 & 1,00 & \\
\hline \multicolumn{7}{|l|}{ IMC pré-gestacional } \\
\hline$<18,5 \mathrm{~kg} / \mathrm{m}^{2}$ & 02 & 8,3 & 13 & 10,8 & 0,64 & 0,58 \\
\hline$\geq 18,5 \mathrm{e}<25 \mathrm{~kg} / \mathrm{m}^{2}$ & 14 & 58,3 & 64 & 53,3 & 1,00 & \\
\hline$\geq 25 \mathrm{e}<30 \mathrm{~kg} / \mathrm{m}^{2}$ & 05 & 20,8 & 35 & 29,2 & 0,28 & $0,05^{\star}$ \\
\hline$\geq 30 \mathrm{~kg} / \mathrm{m}^{2}$ & 03 & 12,5 & 08 & 6,7 & 1,85 & 0,41 \\
\hline
\end{tabular}

${ }^{\star}$ Nível de significância estatística: $\mathrm{p} \leq 0,05 .{ }^{* \star}$ Teste Exato de Fischer. $\ddagger$ Teste ${ }_{\mathrm{x}}{ }^{2}$ de Pearson.

Fonte: Pesquisa de Campo, 2015.

Foi detectado que as mulheres do grupo caso, quando comparadas ao grupo controle, apresentaram frequência de consultas de pré-natal inferior a seis $(58,3 \%$ versus $36,7 \%$ ), de IMC pré-gestacional maior ou igual a $30 \mathrm{~kg} / \mathrm{m}^{2}(12,5 \%$ versus $6,7 \%)$, de etilismo ( $50 \%$ versus $30 \%)$ e medicamento abortivo ( $16,7 \%$ versus $4,2 \%)$. Tais diferenças mostraram associação estatisticamen- te significante $(\mathrm{p}<0,05)$. Observou-se que a chance de crianças nascerem com BPN/ prematuras é 2,94 vezes maior para as mães que fizeram menos de seis consultas no pré-natal; 7,9 vezes maior nas puérperas que consumiram álcool durante a gestação e 5,05 vezes maior para aquelas que usaram medicamento abortivo na gestação. Por sua vez, o IMC pré-gestacional das mães entre 
25 e $30 \mathrm{~kg} / \mathrm{m}^{2}$ revelou-se como fator protetor da ocorrência de BPN/prematuridade (OR: 0,28; $\mathrm{p}=0,05)$ (Tabela 1$)$.

\section{DISCUSSÃo}

Ressalta-se a significativa incidência de BPN e prematuridade $(16,7 \%)$ na população estudada, estando esses valores acima da média nacional $(8,1 \%$ de BPN e $6,5 \%$ de partos prematuros) apresentada no IDB e SINASC, respectivamente. Esses valores são preocupantes, uma vez que estes são os fatores mais importantes na determinação da morbimortalidade neonatal. ${ }^{12-15}$

A gravidez na adolescência é um grave problema de saúde, devendo ser entendida como de risco e vulnerabilidade. ${ }^{16,17}$ No entanto, em caso de gestante de idade mais elevada, é esperada uma maior exposição a doenças crônicas, como hipertensão arterial sistêmica, pré-eclâmpsia, anemia, rotura prematura de membranas, além de diversos fatores de risco potenciais. ${ }^{18}$

No presente estudo, a baixa escolaridade não se mostrou associada a uma maior chance de BPN e de prematuridade, confirmando os achados de Siqueira et al. e Vettore e Lamarca. ${ }^{19,20}$ Entretanto, a frequência da ocupação materna durante a gestação no grupo controle $(32,5 \%)$ foi maior quando comparado aos casos (25\%). Porém, não foram detectadas diferenças relevantes, provavelmente dado o baixo poder do estudo.

O parto por cesariana, por sua vez, não mostrou ser significativamente relacionado com o BPN e a prematuridade, mas vale destacar que este foi mais frequente que o parto natural no grupo controle que no grupo caso ( $56 \%$ versus $43 \%$ ). No entanto, estudos prévios encontraram tal associação. ${ }^{12,21}$

Neste estudo, o parto por cesariana representou 53,5\% dos partos na amostra, o que está muito acima do preconizado pela Organização Mundial de Saúde (OMS), que recomenda que esse procedimento seja executado, no máximo, em 15\% dos nascimentos. ${ }^{16} \mathrm{O}$ valor encontrado está de acordo com outros estudos que apresentam números alarmantes de cesarianas, sendo o método operatório responsável por $52 \%$ do total de partos no Brasil e $46 \%$ na rede pública. ${ }^{22}$ Outro fator de risco citado na literatura refere-se à história de $\mathrm{RN}$ com baixo peso em gestação prévia ${ }^{17}$ e converge com os achados deste estudo.

Embora já seja estabelecido que o etilismo materno durante a gestação possa acarretar perda expressiva do crescimento fetal, ${ }^{23} 33,3 \%$ das puérperas investigadas consumiram álcool durante a gravidez, sendo que $50 \%$ destas faziam parte do grupo caso. Observou-se que a chance de crianças nascerem com BPN/prematuras é 7,9 vezes maior nas puérperas que consumiram álcool durante a gestação.

Os resultados demonstraram que as puérperas expostas ao pré-natal inadequado apresentaram quase três vezes mais chance de terem $\mathrm{RN}$ com peso menor que $2500 \mathrm{~g}$ e parto prematuro, e que a frequência desses eventos ocorreram em mulheres com idade menor ou igual a 25 anos, refletindo significativa associação $(p=0,02)$. Verificou-se que menos de seis consultas aumentou a chance para a ocorrência de BPN/prematuridade, o que converge com o estudo de Vohr et al. ${ }^{24}$ Contudo, Barros et al. ${ }^{18}$ relatam que sete consultas ainda são insuficientes para prevenir RN com baixo peso. 
A influência do acompanhamento pré-natal na prevenção de desfechos gestacionais adversos tem sido estudada na maioria das pesquisas disponíveis na literatura especializada e tem empregado indicadores quantitativos para o estudo de tal relação. ${ }^{21}$ No entanto, autores têm ressaltado a possibilidade de que vieses de seleção (maneira como são escolhidos os sujeitos pertencentes ao estudo) e o modo de organização de serviços de saúde devam ser considerados, visto que os mesmos podem conduzir a efeito de confundimento da associação protetora de adequado pré-natal.

Infecções crônicas da gestante têm efeito negativo no peso ao nascer. ${ }^{25} \mathrm{~A}$ incidência de pré-eclâmpsia, diabetes gestacional, rotura prematura das membranas ovulares e hemorragias periparto, são algumas das complicações obstétricas mais frequentes entre mulheres acima dos 35 anos de idade. Uma revisão de literatura aponta que de $6,0 \%$ a $21,5 \%$ das mulheres com idade avançada apresentaram trabalho de parto prematuro, além de haver uma diminuição na frequência de parto normal com o avançar da idade, sendo a cesariana quatro vezes mais prevalente nessa faixa etária. ${ }^{26}$

A associação entre fatores sociodemográficos e peso ao nascer tem sido destacada na literatura, o que pode ser devido à íntima relação das causas sociais com a desnutrição e infecções. ${ }^{5,9,10}$ Observou-se que o IMC pré-gestacional das mães entre 25 e $30 \mathrm{~kg} / \mathrm{m}^{2}$ revelou-se como fator protetor da ocorrência de BPN/prematuridade (OR: 0,28; $\mathrm{p}=0,05$ ).

\section{CONSIDERAÇõeS FINAIS}

Considerando que o BPN é uma condição que serve de alerta de que algo errado aconteceu durante o período gestacional, ${ }^{27}$ os achados deste estudo poderão favorecer na identificação de novas abordagens do profissional em relação às gestantes. Essas poderão ser favorecidas, pois um melhor entendimento do seu estado de saúde é fundamental para a adoção de condutas mais adequadas e prevenção de agravos no período gestacional.

Dessa forma, a identificação dos fatores de risco do BPN e da prematuridade é relevante para a saúde pública, possibilitando subsídios para o planejamento de estratégias de enfrentamento desse agravo.

Quanto às limitações desta investigação, devem ser observadas as do próprio delineamento metodológico escolhido, como o viés de memória, devido ao seu caráter retrospectivo, o viés de seleção dos grupos, além do possível constrangimento da puérpera durante a entrevista. No entanto, acreditamos que haja necessidade de um maior investimento investigativo sobre a temática com arranjos metodológicos robustos e com maior poder de análise, a fim de que os fatores de risco associados a esses eventos sejam melhor esclarecidos. 


\section{REFERÊNCIAS}

1. Franciscatto LHG, Pasqua MD, Tolotti GK, Rossetto C., Argent C., Pinheiro JM. Delineamento do perfil epidemiológico de puérperas e recém-nascidos. Rev enferm UFPE on line 2014; 8(5): 1.149-56.

2. Andrade SM, Soares DA, Tiemi M., Souza RKT, Mathias, TAF, Iwakura MLH et al. Condições de vida e mortalidade infantil no Estado do Paraná, Brasil, 1997/2001. Cad Saúde Pública 2010: 22(1), 181-189.

3. Brasil. Ministério da Saúde. Secretaria de Atenção à Saúde. Departamento de Ações Programáticas Estratégicas. Atenção à Saúde do Recém-Nascido: Guia para os profissionais de saúde, 2. ed. Brasília-DF: Ministério da Saúde; 2012.

4. Victora CG, Aquino EML, Leal MC, Monteiro CA, Barros FC, Szwarcwald CL. Maternal and child health in Brazil: progress and challenges. Lancet 2011; 377(9780): 1.863-76. http://dx.doi. org/10.1016/S0140-6736(11)60138-4

5. Cruz SS, Costa M. da CN, Gomes-Filho IS, Barreto ML. Periodontal therapy for pregnant women and cases of low birthweight: An intervention study. Pediatr Int. 2010; 52(1): 57-64.

6. Malta DC, Duarte EC, Escalante JJC, Almeida MF, Sardinha LMV, Macário EM. Mortes evitáveis em menores de um ano, Brasil, 1997 a 2006: contribuições para a avaliação de desempenho do Sistema Único de Saúde. Cad Saúde Pública 2010: 26(3): 481-91.

7. Rojas PFB, Carminatti APS, Hafemann F, Stein AC, FranciscoCC. Fatores maternos preditivos de baixo peso ao nascer: um estudo caso-controle. Arq. Catarin. Med. 2013; 42(1): 68-75.

8. Ebersole JL, Novak MJ, Michalowicz BS, Hodges JS, Steffen MJ, Ferguson JE, Diangelis A., Buchanan W., Mitchell DA, Papapanou PN. Sistemic immune responses in pregnancy and periodontitis: relationship to pregnancy outcomes in the obstetrics and periodontal therapy (OPT) study. J Periodontol. 2009; 80(6): 953-60.

9. Geib LTC, Fréu CM, Brandão M., Nunes ML. Determinantes sociais e biológicos da mortalidade infantil em coorte de base populacional em Passo Fundo, Rio Grande do Sul. Ciênc Saúde Coletiva. 2010; 15(2): 363-70.

10. Ramos HAC, Cuman RKN. Fatores de risco para prematuridade: pesquisa documental. Esc Anna Nery Rev Enferm. 2009 abr./jun.; 13 (2): 297-304.

11. BRASIL. Conselho Nacional de Saúde. Resolução n. 466, de 12 de dezembro de 2012. Brasília, 2012.

12. Barros F., Matijasevich A., Silveira M. Estudo “Prematuridade e suas possíveis causas”. Unicef 2013.

13. Silveira MF, Santos IS, Barros AJD, Matijasevich A., Barros FC, Victora CG. Aumento da prematuridade no Brasil: revisão de estudos de base populacional. Rev Saúde Pública 2008; 42(5): 957-64.

14. IBGE. Indicadores Sociodemográficos e de Saúde no Brasil. Rio de Janeiro: 2009.

15. Ministério da Saúde (BR). Secretaria de Atenção à Saúde. Departamento de Ações Programáticas Estratégicas. Manual de vigilância do óbito infantil e fetal e do comitê de prevenção do óbito infantil e fetal. Brasília: Ministério da Saúde; 2009.

16. Silva JLP, Surita FGC. Gravidez na adolescência: situação atual. Rev Bras Ginecol Obstet. 2012; 34(8): 347-50.

17. World Health Organization. Major causes of death in neonates and children under five GLOBAL 2008 (revised). The World Health Organization Statistic [homepage da Internet] 2011 [acesso em 20/01/2017]. Disponível em: http://www.who.int/gho/publications/world_health_statistics/EN_WHS08_Full.pdf. 
18. Barros FC, Victora CG, Matijasevich A., Santos IS, Horta BL, Silveira MF et al. Preterm births, low birth weight, and intrauterine growth restriction in three birth cohorts in Southern Brazil: 1982, 1993 and 2004. Cad Saúde Pública 2008; 24 Suppl. 3:S390-8.

19. Siqueira CGDPC, Monteiro BAA, Toste SACBF, Capelli CJDCS. Baixo peso ao nascer: fatores socioeconômicos, assistência pré-natal e nutricional - uma revisão. Revista Augustus. 2010; fev. 14(29).

20. Vettore M., Lamarca G. Atenção pré-natal no Brasil: uma questão de oferta, acesso ou de escolaridade materna? Rio de Janeiro: Portal DSS Brasil, 2012.

21. Cruz RSBLC, Caminha MFC, Batista Filho M. Aspectos históricos, conceituais e organizativos do pré-natal. Revista Brasileira de Ciências da Saúde. 2014; 18(1): 87-94.

22. Padilha JF, Torres RPP, Gasparetto A., Farinha LB, Mattos KM. Parto e idade: características maternas do Estado do Rio Grande do Sul. Saúde (Santa Maria) 2013; 39(2): 99-108.

23. Freire K., Padilha PC, Saunders C. Fatores associados ao uso de álcool e cigarro na gestação. Rev Bras Ginecol Obstet. 2009; 31(7): 335-41.

24. Vohr BR, Tyson JE, Wright LL, Perritt RL, Li L, Poole WK. Maternal age, multiple birth, and extremely low birth weight infants. J Pediatr. 2009; 154(4): 498-503.e2.

25. Beck S1, Wojdyla D., Say L., Betran AP, Merialdi M., Requejo JH, Rubens C., Menon R., Van Look PF. The worldwide incidence of preterm birth: a systematic review of maternal mortality and morbidity. Bull World Health Organ. 2010; 88(1): 31-8.

26. Gonçalves ZR, Monteiro DLM. Complicações maternas em gestantes com idade avançada. Feminina 2012; 40(5): 275-9.

27. Carvalho SS, Coelho JMF, Soares DAB, Mariola E. Fatores maternos para o nascimento de recém-nascidos com baixo peso e prematuros: estudo caso-controle. Rev Ciência e Saúde mai./ ago. 2016; 9(2): 76-82.

\section{DADOS Do AUTOR}

\section{Silas Santos Carvalho}

Mestrando em Saúde Coletiva na Universidade Estadual de Feira de Santana. Pós-graduado em Saúde da Mulher, Enfermagem Obstétrica e Bacharelado em Enfermagem pela Faculdade Anísio Teixeira de Feira de Santana. Feira de Santana/BA - Brasil. ssc.academico@hotmail.com

\section{Julita Maria Freitas Coelho}

Doutora em Saúde Pública.

Dentista. Professora na Faculdade Anísio Teixeira e da Universidade Estadual de Feira de Santana.

Submetido em: 18-2-2017

Aceito em: 5-4-2017 